\title{
A New Characterization of Totally Umbilical Hypersurfaces in de Sitter Space
}

\author{
Linfen $\mathrm{Cao}^{1}$, Guodong $\mathrm{Xu}^{2}$, Zhaohui Dai ${ }^{3}$ \\ ${ }^{1}$ College of Mathematics and Information Science, Henan Normal University, Xinxiang, China \\ ${ }^{2}$ School of Mathematics and Statistics, Nangyang Normal University, Nangyang, China \\ ${ }^{3}$ Department of Computer Science, Henan Normal University, Xinxiang, China \\ Email: caolf2010@yahoo.com
}

Received November 4, 2013; revised December 4, 2013; accepted December 11, 2013

Copyright (C) 2014 Linfen Cao et al. This is an open access article distributed under the Creative Commons Attribution License, which permits unrestricted use, distribution, and reproduction in any medium, provided the original work is properly cited. In accordance of the Creative Commons Attribution License all Copyrights (C) 2014 are reserved for SCIRP and the owner of the intellectual property Linfen Cao et al. All Copyright (C) 2014 are guarded by law and by SCIRP as a guardian.

\section{ABSTRACT}

It is shown that a compact spacelike hypersurface which is contained in the chronological future (or past) of an equator of de Sitter space is a totally umbilical round sphere if the $k$ th mean curvature function $H_{k}$ is a linear combination of $H_{k+1}, \cdots, H_{n}$. This is a new angle to characterize round spheres.

\section{KEYWORDS}

\section{de Sitter Space; Spacelike Hypersurface; Higher Order Mean Curvatures}

\section{Introduction}

Let $R_{1}^{n+2}$ be the $(n+2)$-dimensional Lorentz-Minkowski space, namely, the real vector space $R^{n+2}$ endowed with the Lorentzian inner product $\langle$,$\rangle given by$

$$
\langle v, w\rangle=\sum_{i=1}^{n+1} v_{i} w_{i}-v_{n+2} w_{n+2}, v=\left(v_{1}, \cdots, v_{n+2}\right), w=\left(w_{1}, \cdots, w_{n+2}\right) \in R^{n+2} .
$$

Then the $n$-dimensional de Sitter space is defined by $S_{1}^{n+1}=\left\{x \in R_{1}^{n+2} \mid\langle x, x\rangle=1\right\}$. It is well known that, for $n \geq 2$ the de Sitter space $S_{1}^{n+1}$ is the standard simply connected Lorentzian space form of positive constant sectional curvature. A smooth immersion $\psi: M^{n} \rightarrow S_{1}^{n+1} \subset R_{1}^{n+2}$ of an $n$-dimensional connected manifold $M^{n}$ is said to be a spacelike hypersurface if the induced metric via $\psi$ is a Riemannian metric on $M^{n}$, which, as usual, is also denoted by $\langle$,$\rangle .$

The interest for the study of spacelike hypersurfaces in de Sitter space is motivated by the fact that such hypersurfaces exhibit nice Bernstein-type properties. In 1977, Goddard [1] conjectured that the only complete spacelike hypersurfaces with constant mean curvature in $S_{1}^{n+1}$ should be the totally umbilical ones. This conjecture motivated the work of an important number of authors who considered the problem of characterizing the totally umbilical spacelike hypersurfaces of de Sitter space. In [2], Montiel showed that the only compact spacelike hypersurfaces in $S_{1}^{n+1}$ with constant mean curvature $H_{1}$ were the totally umbilical round spheres. More recently, Cheng and Ishikawa [3] have shown that the totally umbilical round spheres are the only compact spacelike hypersurfaces in de Sitter space with constant scalar curvature $S<n(n-1)$.

The natural generalization of mean and scalar curvature for a spacelike hypersurface in de Sitter space are the $k$ th mean curvature $H_{k}$ for $k=1, \cdots, n$. Actually, $H_{1}$ is the mean curvature and $H_{2}$ is, up to a constant, the scalar curvature of the hypersurface. In [4], Aledo, jointly with Alias and Romero, developed some integral formulas for compact spacelike hypersurfaces in $S_{1}^{n+1}$ and applied them in order to characterize the totally umbilical round spheres of $S_{1}^{n+1}$. 
Theorem 1([4], Theorem 7) Let $\psi: M^{n} \rightarrow S_{1}^{n+1} \subset R_{1}^{n+2}$ be a compact spacelike hypersurface in de Sitter space which is contained in the chronological future (or past) of an equator of $S_{1}^{n+1}$. If $H_{k}$ is constant for some $k, 1 \leq k \leq n$, then $M^{n}$ is a totally umbilical round sphere.

Since $H_{0}=1$ by definition, the result above can be read as follows: if $H_{k} / H_{0}$ is constant for some $k, 1 \leq k \leq n$, then $M^{n}$ is a totally umbilical round sphere. In [5], Alias extended Theorem 1 in the following way.

Theorem 2 ([5]) Let $\psi: M^{n} \rightarrow S_{1}^{n+1} \subset R_{1}^{n+2}$ be a compact spacelike hypersurface in de Sitter space which is contained in the chronological future (or past) of an equator of $S_{1}^{n+1}$. If $H_{l}$ does not vanish on $M^{n}$ and the ratio $H_{k} / H_{l}$ is constant for some $k, l, 1 \leq l<k \leq n$, then $M^{n}$ is a totally umbilical round sphere.

In [6] the authors considered that $H_{k}$ is the linear combination of $H_{1}, \cdots, H_{k-1}$, and proved:

Theorem 3 ([6]) Let $\psi: M^{n} \rightarrow S_{1}^{n+1} \subset R_{1}^{n+2}$ be a compact spacelike hypersurface in de Sitter space which is contained in the chronological future (or past) of an equator of $S_{1}^{n+1}$. If there are nonnegative constants $C_{1}, C_{2}, \cdots, C_{l-1}$, at least one $C_{i}$ is positive, such that $H_{l}=\sum_{i=1}^{l-1} C_{i} H_{i}$ holds on $M^{n}$, then $M^{n}$ is a totally umbilical round sphere.

In this paper, we will show another characterization of totally umbilical round sphere, which extends Theorems 1 and 2 above.

Theorem 4 Let $\psi: M^{n} \rightarrow S_{1}^{n+1} \subset R_{1}^{n+2}$ be a compact spacelike hypersurface in de Sitter space which is contained in the chronological future (or past) of an equator of $S_{1}^{n+1}$. If $H_{n}$ does not vanish on $M^{n}$,

- for some fixed $k, 1 \leq k \leq n-1$, there exist constants $C_{j} \geq 0$ such that $H_{k}=\sum_{j=k+1}^{n} C_{j} H_{j}$ on $M^{n}$, then $M^{n}$ is a totally umbilical round sphere.

- there are $n-1$ constants $C_{j} \geq 0$ such that $H_{0}=\sum_{j=1}^{n-1} C_{j} H_{j}$ on $M^{n}$, then $M^{n}$ is a totally umbilical round sphere.

\section{Remark.}

- Note in some special cases the condition $H_{n}$ does not vanish should can be dropped, for examples, only one coefficient $C_{j}>0$ case. However in general cases we can not drop it now.

- The corresponding theorem characterizes ellipsoids also holds in affine differential geometry.

\section{Preliminaries}

Throughout this paper we will deal with compact spacelike hypersurfaces in de Sitter space. Recall that every compact spacelike hypersurfaces $M^{n}$ in $S_{1}^{n+1}$ is diffeomorphic to an $n$-sphere [4] and, in particular, it is orientable. Then, there exists a timelike unit normal field $N$ globally defined on $M^{n}$. We will refer to $N$ as the Gauss map of the immersion and we will say that $M^{n}$ is oriented by $N$.

We will denote by $A: \chi(M) \rightarrow \chi(M)$ the shape operator of $M^{n}$ in $S_{1}^{n+1}$ with respect to $N$, which is given by

$$
A(X)=-d N(X) .
$$

Associated to the shape operator of $M^{n}$ there are $n$ algebraic invariants, which are the elementary symmetric functions $\sigma_{k}$ of its principal curvatures $k_{1}, \cdots, k_{n}$, given by

$$
\sigma_{k}\left(k_{1}, \cdots, k_{n}\right)=\sum_{i_{1}<\cdots<i_{k}} k_{i_{1}} \cdots k_{i_{k}}, \quad 1 \leq k \leq n .
$$

The $k$ th mean curvature $H_{k}$ of the spacelike hypersurfaces is then defined by

$$
\left(\begin{array}{l}
n \\
k
\end{array}\right) H_{k}=(-1)^{k} \sigma_{k}\left(k_{1}, \cdots, k_{n}\right)=\sigma_{k}\left(-k_{1}, \cdots,-k_{n}\right) \text {. }
$$

When $k=1, \quad H_{1}=-(1 / n) \operatorname{tr}(A)$ is the mean curvature of $M^{n}$. On the other hand, when $k=n$, $H_{n}=(-1)^{n} \operatorname{det}(A)$ defines the Gauss-Kronecker curvature of the spacelike hypersurface, and for $k=2, H_{2}$ is, up to a constant, the scalar curvature $S$ of $M^{n}$, since $S=n(n-1)\left(1-H_{2}\right)$ (for details see [4]).

The proof of our theorem makes an essential use of the following integral formulas for compact spacelike hypersurfaces in $S_{1}^{n+1}$, which is developed in [4].

Lemma 5 (Minkowski formulas) Let $\psi: M^{n} \rightarrow S_{1}^{n+1} \subset R_{1}^{n+2}$ be a compact spacelike hypersurface immersed into de Sitter space and let $a \in R_{1}^{n+2}$ a fixed arbitrary vector. For each $r=0, \cdots, n-1$ the following formula holds: 


$$
\int_{M}\left(-H_{r}\langle a, \psi\rangle+H_{r+1}\langle a, N\rangle\right) \mathrm{d} V=0,
$$

where $\mathrm{d} V$ is the $n$-dimensional volume element of $M^{n}$ with respect to the induced metric and the chosen orientation.

\section{Proof of the Theorem 4}

Let us assume, for instance, that the hypersurface $\psi: M^{n} \rightarrow S_{1}^{n+1} \subset R_{1}^{n+2}$ is contained in the future of the equator determined by a unit timelike vector $a \in R_{1}^{n+2}$ (the case of the past is similar). That means that

$$
\psi(M) \subset\left\{x \in S_{1}^{n+1}:\langle a, x\rangle<0\right\} .
$$

Let us orient $M^{n}$ by the Gauss map $N$ which is in the same time-orientation as $a$, so that $\langle a, N\rangle \leq-1<0$. Since the height function $\langle a, \psi\rangle$ is negative on $M^{n}$, by compactness there exists a point $p_{0} \in M$ where it attains its maximum

$$
\left\langle a, \psi\left(p_{0}\right)\right\rangle=\max _{p \in M}\langle a, \psi(p)\rangle<0 .
$$

Therefore, its gradient vanishes at that point, $\nabla\langle a, \psi\rangle\left(p_{0}\right)=0$, and its Hessian satisfies

$$
\nabla^{2}\langle a, \psi\rangle\left(p_{0}\right)(v, w)=-\left\langle a, \psi\left(p_{0}\right)\right\rangle\langle v, w\rangle-\left\langle a, N\left(p_{0}\right)\right\rangle\left\langle A_{p_{0}}(v), w\right\rangle \leq 0
$$

for all $v, w \in T_{p_{0}} M \quad$ (for the details see the proof of Theorem 7 in [4]). On the other hand, since

$$
\langle a, N\rangle^{2}=1+\langle a, \psi\rangle^{2}+|\nabla\langle a, \psi\rangle|^{2}
$$

and

$$
\nabla\langle a, \psi\rangle\left(p_{0}\right)=0
$$

then

$$
-\left\langle a, N\left(p_{0}\right)\right\rangle=\sqrt{1+\left\langle a, \psi\left(p_{0}\right)\right\rangle^{2}} .
$$

Therefore, choosing $\left\{e_{1}, \cdots, e_{n}\right\}$ a basis of principal directions at the point $p_{0}$ we conclude that

$$
k_{i}\left(p_{0}\right) \leq \frac{\left\langle a, \psi\left(p_{0}\right)\right\rangle}{\sqrt{1+\left\langle a, \psi\left(p_{0}\right)\right\rangle^{2}}}<0
$$

for each $i=1, \cdots, n$. In particular, $H_{j}\left(p_{0}\right)(1 \leq j \leq n)$ are positive. The mean curvature functions $H_{n}$ is positive on $M^{n}$ (recall that $H_{n}$ does not vanish on $M^{n}$ by assumption). Therefore, from the proof of Lemma 1 in [7] and taking into account the sign convention in our definition of the higher order mean curvature, it follows that every $H_{j}$ is positive for $j=1, \cdots, n$ and

$$
\frac{H_{1}}{H_{0}} \geq \frac{H_{2}}{H_{1}} \geq \cdots \geq \frac{H_{n}}{H_{n-1}},
$$

with equality at any stage only at umbilical points.

Let us start proving the first statement of Theorem 4. Using

$$
H_{k}=\sum_{j=k+1}^{n} C_{j} H_{j}
$$

and the Minkowski formulae, we have

$$
\int_{M} H_{k-1}\langle a, \psi\rangle \mathrm{d} V=\int_{M} H_{k}\langle a, N\rangle \mathrm{d} V=\sum_{j=k+1}^{n} C_{j} \int_{M} H_{j}\langle a, N\rangle \mathrm{d} V=\sum_{j=k+1}^{n} C_{j} \int_{M} H_{j-1}\langle a, \psi\rangle \mathrm{d} V
$$

That is,

$$
\int_{M}\langle a, \psi\rangle\left(H_{k-1}-\sum_{j=k+1}^{n} C_{j} H_{j-1}\right) \mathrm{d} V=0 .
$$


Now we claim that

$$
H_{k-1}-\sum_{j=k+1}^{n} C_{j} H_{j-1} \leq 0
$$

on $M$, with equality if and only if $k_{1}=\cdots=k_{n}$. Assume that (3) is true. Then, since $\langle a, \psi\rangle<0$ on $M$, we conclude that

$$
H_{k-1}-\sum_{j=k+1}^{n} C_{j} H_{j-1} \equiv 0,
$$

which implies that $M$ is an totally umbilical round sphere.

It remains to prove (3). Using the assumption of theorem 4, that is $H_{k}=\sum_{j=k+1}^{r} C_{j} H_{j}$ and (2), we have

$$
H_{k-1}-\sum_{j=k+1}^{n} C_{j} H_{j-1}=\sum_{j=k+1}^{n} C_{j}\left(\frac{H_{k-1}}{H_{k}} H_{j}-H_{j-1}\right) \leq 0 \text {. }
$$

Now we prove the second statement. Using

$$
H_{0}=\sum_{j=1}^{n-1} C_{j} H_{j}
$$

and the Minkowski formulae, we have

$$
\int_{M} H_{1}\langle a, N\rangle \mathrm{d} V=\int_{M} H_{0}\langle a, \psi\rangle \mathrm{d} V=\sum_{j=1}^{n-1} C_{j} \int_{M} H_{j}\langle a, \psi\rangle \mathrm{d} V=\sum_{j=1}^{n-1} C_{j} \int_{M} H_{j+1}\langle a, N\rangle \mathrm{d} V
$$

That is,

$$
\int_{M}\langle a, N\rangle\left(H_{1}-\sum_{j=1}^{n-1} C_{j} H_{j+1}\right) \mathrm{d} V=0
$$

Now we claim that

$$
H_{1}-\sum_{j=1}^{n-1} C_{j} H_{j+1} \geq 0
$$

on $M$, with equality if and only if $k_{1}=\cdots=k_{n}$. Assume that (5) is true. Then, since $\langle a, N\rangle<0$ on $M$, we conclude that

$$
H_{1}-\sum_{j=1}^{n-1} C_{j} H_{j+1} \equiv 0,
$$

which implies that $M$ is an totally umbilical round sphere. It remains to prove (5). As in the first proof, using the assumption of theorem 4, that is $H_{0}=\sum_{j=1}^{n-1} C_{j} H_{j}$, and (2) we known

$$
H_{1}-\sum_{j=1}^{n-1} C_{j} H_{j+1}=\sum_{j=1}^{n-1} C_{j}\left(\frac{H_{1}}{H_{0}} H_{j}-H_{j+1}\right) \geq 0 .
$$

This completes the proof of the Theorem 4.

\section{Funding}

This work is supported by grant (No.U1304101 and 11171091) of NSFC and NSF of Henan Province (No.132300410141).

\section{REFERENCES}

[1] A. J. Goddard, “Some Remarks on the Existence of Spacelike Hypersurfaces of Constant Mean Curvature,” Mathematical Proceedings of the Cambridge Philosophical Society, Vol. 82, 1977, pp. 489-495. http://dx.doi.org/10.1017/S0305004100054153

[2] S. Montiel, “An Integral Inequality for Compact Spacelike Hypersurfaces in de Sitter Space and Applications to the Case of 
Constant Mean Curvature,” Indiana University Mathematics Journal, Vol. 37, No. 4, 1988, pp. 909-917. http://dx.doi.org/10.1017/S0305004100054153

[3] Q.-M. Cheng and S. Ishikawa, "Spacelike Hypersurfaces with Constant Scalar Curvature,” Manuscripta Mathematica, Vol. 95, No. 4, 1998, pp. 499-505. http://dx.doi.org/10.1007/s002290050043

[4] J. A. Aledo, L. J. Alias and A. Romero, "Integral Formulas for Compact Space-Like Hypersurfaces in de Sitter Space: Applications to the Case of Constant Higher Mean Curvature,” Journal of Geometry and Physics, Vol. 31, No. 2-3, 1999 , pp. $195-208$. http://dx.doi.org/10.1016/S0393-0440(99)00008-X

[5] L. J. Alias and S.-E. Koh, "Remarks on Compact Spacelike Hypersurfaces in de Sitter Space with Constant Higher Order Mean Curvature,” Journal of Geometry and Physics, Vol. 39, No. 1, 2001, pp. 45-49. http://dx.doi.org/10.1016/S0393-0440(00)00073-5

[6] S.-E. Koh and M. S. Yoo, “A Characterization of Totally Umbilical Hypersurfaces in de Sitter Space,” Journal of Geometry and Physics, Vol. 51, No.1, 2004, pp. 34-39. http://dx.doi.org/10.1016/j.geomphys.2003.09.006

[7] S. Montiel and A. Ros, “Compact Hypersurfaces: The Alexandrov Theorem for Higher Order Mean Curvatures,” In: B. Lawson and K. Tenenblat, Eds., Differential Geometry, Longman, Essex, 1991, pp. 279-296.

[8] K. Akutagawa, “On Spacelike Hypersurfaces with Constant Mean Curvature in the de Sitter Space,” Mathematische Zeitschrift, Vol. 196, No. 1, 1987, pp. 13-19. http://dx.doi.org/10.1007/BF01179263

[9] L. J. Alias and A. G. Colares, “A Further Characterization of Ellipsoids,” Results in Mathematics, Vol. 48, No.1-2, 2005, pp. 1-8. http://dx.doi.org/10.1007/BF03322891

[10] H. Li, “Global Rigidity Theorems of Hypersurfaces,” Arkiv for Matematik, Vol. 35, No. 2, 1997, pp. 327-351.

[11] J. Ramanathan, “Complete Spacelike Hypersurfaces of Constant Mean Curvature in de Sitter Space,” Indiana University Mathematics Journal, Vol. 36, No. 2, 1987, pp. 349-359. http://dx.doi.org/10.1512/iumj.1987.36.36020

[12] Y. Zheng, “On Space-Like Hypersurfaces in the de Sitter Space,” Annals of Global Analysis and Geometry, Vol.13, No. 4, 1995, pp. 317-321. http://dx.doi.org/10.1007/BF00773403

[13] Y. Zheng, "Space-Like Hypersurfaces with Constant Scalar Curvature in the de Sitter Space,” Differential Geometry and Its Applications, Vol. 6, No. 1, 1996, pp. 51-54. http://dx.doi.org/10.1016/0926-2245(96)00006-X 